\title{
Editorial
}

\section{Implementing a DNR policy: promise and perils}

Many North American hospitals have now developed institutional policies to govern the use of "Do-notresuscitate" (DNR) orders. Although the policies adopted vary in several respects, a broad consensus seems to have emerged about the sort of guidelines which are appropriate. However, the transition from theory to practice is not always smooth. The best policy in the world may produce poor results if it is misunderstood by those whose actions it is intended to guide, or if there is no agreement about its interpretation, or if it is filed away alongside other institutional policy statements. For this reason, the medical community should welcome the investigation performed by Webster et al. and attend carefully to their findings and recommendations.

\section{When "treatment" ceases to be "therapeutic"}

First we should consider a semantic point which may affect how DNR decisions are viewed. Webster et al. describe the decision to withhold cardio-pulmonary resuscitative measures from patients within an intensive care unit "an important limitation of therapy." The unwary might infer from this that CPR is always "therapeutic," and its refusal or denial is a restriction of treatment. But in circumstances where CPR would produce no benefit for the patient (or when it would actually produce an adverse net balance of good over bad results), then it would not be therapeutic and its restricted use should not be seen as a "limitation of therapy."

This point is worth making to counteract the excessively curative orientation which pervades the thinking of many health professionals. Amongst physicians, in particular, there appears to be an understandable, but regrettable, bias towards aggressive treatment.' Those who require overwhelming evidence before they will recognize that death is inevitable may sometimes, inadvertently, be guilty of cruelty.

From the Centre for Professional and Applied Ethics, University of Manitoba, University College, 500 Dysart Road, Winnipeg, Manitoba, R3T 2M8.

\section{Charting decisions}

Webster et al. cite a recent American study (Ref. \#1) suggesting that DNR orders were almost always documented in the written medical record before the withholding or withdrawal of life support. However, the quoted study investigated DNR decisions at only two intensive care units. Other recent studies suggest, on the contrary, a widespread failure of adequate documentation. ${ }^{2.3}$

One wonders if, in Canadian intensive care units, the DNR orders in patients' charts are also accompanied by (a) an overall treatment plan, (b) a list of the specific treatments or therapies (other than CPR) which are to be given or withheld, (c) a clearly stated rationale, and (d) a statement of the patient's wishes with respect to treatment (or, in the case of an incompetent patient, a statement of the family's view of what the patient's wishes would have been).

These questions are important for several reasons. When patients decide that they do not want all-out aggressive treatment to prolong their lives, they may not be choosing complete medical non-intervention. At the least, they may expect to receive relief from pain, and the care and comfort necessary to reassure them that they have not been abandoned. It is not uncommon to find physicians who declare that they do not allow a DNR decision to be charted because they fear that "hopeless" patients may receive a reduced level of care and comfort from doctors and nurses.

If medical schools were to incorporate the management of pain and discomfort as a more central part of their undergraduate curriculum, this might counterbalance the prevailing emphasis on curative methods. Moreover, a palliative care orientation is enhanced in hospitals which provide transitional spaces for patients whose disease can be neither cured nor controlled.

Going half-hog rather than whole hog or no hog at all In their "Discussion" Webster et al. note that "the extent to which physicians and nurses equated the DNR order with limiting treatment generally was unexpected." They recommend that a DNR order not be viewed as equivalent 
to an order for the withdrawal of all treatment. This deserves to be heavily underscored. In order to minimize the dangers of inconsistency, confusion, and misunderstanding amongst the rotating teams of hospital doctors and nurses, it is crucial that the paient's chart clearly indicates just which interventions are to be excluded. It might be helpful if "Procedural Guidelines" required that the goals of treatment and the wishes of the patient and/or his family, also be written in the chart. Such written guidance is invaluable to staff covering an emergency and may spare the patient from inappropriate interventions. $^{2-4}$

\section{The patient as decision-maker}

The authors were not surprised to discover that $22 / 25$ patients deemed unsuitable for cardiopulmonary resuscitation "lacked decision-making capacity," though they later observed that "several nurses expressed the view that consideration of the DNR order had been delayed and could have been discussed with patients earlier, when their mental status was not seriously compromised."

Disappointingly, the DNR protocol adopted in this report does not invite consultation with the patient about such decisions except "when clinical assessment justifies the writing of a 'no resucitation' order." By this time, most patients were incompetent to exercise their right to autonomy.

The St. Michael's experience is not atypical of North American hospitals. The DNR decisions are commonly made so late that a majority of patients has become incompetent to participate in the consultative process. ${ }^{5}$ If we value the participation of patients in DNR decisionmaking, then there is a need to find effective ways of involving patients before they become too sick, or too stupified by pain-killing drugs, to express their own wishes. In this regard the proposal, that such issues be raised with all patients when they are admitted to hospital, is worth serious consideration.

\section{The family as surrogate decision-maker}

Webster et al. noted that physicians were sometimes concerned about "familial ambivalence" and that physicians, themselves, were uncertain about the "proper" role of families in decisions such as the DNR order or withdrawal of treatment. When families were invited to participate in DNR decisions, they "sometimes felt that they were being placed unfairly in the role of deciding whether a loved one lives or dies." In their analysis of the implementation process, the authors noted that "families were almost always consulted regarding the DNR decision." However, they did not analyse the nature of this consultation, so they were unable to explain whether the familial ambivalence and unease were due to the wrong question(s) being asked.
Perhaps the protocol should be amended to make it clear that the family's view(s) be canvassed only when it is impossible to reconstruct the patient's own wishes or intentions regarding treatment. They should be consulted with regard to the patient's views, attitudes and values, rather than their own.

The role of nursing and allied staff in DNR decisions The authors have indicated that there are important "differences in perception of communication between physicians and nurses." They attribute these differences partly to physician failure to consult directly with nurses and partly to the nursing profession's special perspective on patient care - arising from the bedside nurse's "intense and prolonged patient contact." perception between physicians and nurses, the authors appear to favour the team approach to decision-making and consider that "the implementation of our DNR policy ... led to enhanced communication in the ICU." They do not, however, unequivocally encourage nurses in their role as patient advocates. This is a point of potential conflict which requires further analysis and evaluation.

It would be interesting to know when physicians consulted nurses, and whether they regard nurses as sources of information about the patient's or the family's wishes, or as substitute decision-makers.

\section{Conclusion}

Webster et al. have made an important contribution to our understanding of the realities of DNR decision-making in a Canadian hospital. They conclude that further studies are required to corroborate their positive experience. To this one might add the hope that further investigation will also deepen our understanding of the underlying value issues.

\section{La mise en place d'une politique de non-réanimation : espoir et périls}

Plusieurs hôpitaux nord américains ont maintenant développé des politiques institutionnelles afin de réglementer les ordres de non-réanimation (DNR). Même si les politiques adoptées varient dans plusieurs aspects, un large consensus semble émerger concernant le genre de 
directives qui seraient adéquates. Cependant, la transition de la théorie à la pratique n'est pas toujours facile. La meilleure politique au monde peut produire des résultats médiocres lorsqu'imcomprise par ceux qu'elle désire guider l'action ou par l'absence de consensus sur son interprétation, ou quand elle est classée comme une autre politique institutionnelle. Pour cette raison, la communauté médicale doit s'intéresser à l'investigation faite par Webster $e t$ al. et faire attention à leurs recommendations et trouvailles.

Quand le * traitement " cesse d'être « thérapeutique " On doit premièrement considérer un point sémantique qui peut affecter la façon dont les ordres de nonréanimation sont perçues. Webster et al. décrivent la décision de s'abstenir d'entreprendre des mesures de réanimation cardiopulmonaire à certains patients de l'unité des soins intensifs comme "une limitation importante de la thérapie ". Les non avisés peuvent en déduire qu'étant donné que la réanimation cardiopulmonaire est «toujours thérapeutique ", son retrait ou le refus de son exécution serait une restriction du traitement. Mais, dans les circonstances oû la réanimation cardiopulmonaire ne pourrait produire aucun effet sur le patient (ou quand elle pourrait réellement produire des résultats finaux néfastes en tenant compte des bénéfices versus avantages), elle ne serait pas alors thérapeutique et l'abstention de son exécution ne devrait pas être conçue comme une « limitation de la thérapie».

Ce point est important afin de contrecarrer l'orientation curative excessive qui envahit la pensée de plusieurs professionnels de la santé. Parmi les médecins en particulier, il apparait un biais compréhensible mais regrettable vers une thérapie agressive.' Ceux qui requièrent une évidence écrasante avant d'admettre que la mort est inévitable peuvent quelquefois par inadvertance être coupable de cruauté.

\section{La documentation des décisions}

Webster et al. citent une étude américaine récente (Ref. \#1) suggérant que les ordres de non-réanimation étaient presque toujours documentés dans le dossier médical écrit avant le retrait ou la non exécution du support vital. Cependant, l'étude mentionnée investigué les décisions de non réanimation uniquement dans deux unités de soins intensifs. D'autres études récentes suggèrent, au contraire, un fréquent défaut de documentation adéquate. ${ }^{2.3}$

On se demande si, dans les unités de soins intensifs canadiennes, les ordres de non réanimation dans les dossiers des patients sont aussi accompagnés de a) un plan de traitement global, b) une liste de traitement ou thérapie spécifique (autre que réanimation cardiopulmonaire) qui serait à administrer ou à éviter, c) une rationnelle clairement énoncée, et d) un énoncé des désirs du patient concernant le traitement (ou, dans le cas d'un patient incompétent, un énoncé du point de vue de la famille concemant la volonté propre du patient).

Ces questions sont importantes pour plusieurs raisons. Quand les patients décident qu'ils ne désirent pas un traitement global agressif afin de prolonger leur vie, ils peuvent ne pas vouloir choisir une abstention thérapeutique totale. Pour le moins, ils peuvent s'attendre à recevoir un soulagement de la douleur, le soin et le confort nécessaires pour les rassurer qu'ils ne seront pas abandonnés. Il n'est pas rare de trouver des médecins qui déclarent qu'ils ne désirent pas documenter la décision de non réanimation de peur que leurs patients "sans recours " puissent recevoir une qualité réduite de soins et de confort de la part des autres médecins et personnel infirmier.

Si les facultés de médecine mettaient l'emphase sur le confort et le soulagement de la douleur des patients comme partie essentielle du programme de leurs étudiants, ceci pourrait contrebalancer les méthodes curatives qui sont actuellement dominantes. De plus, une orientation vers des soins palliatifs s'est accrue dans les hôpitaux ce qui fournirait une classe transitionnelle pour les patients dont la maladie ne peut être ni guérie ni contrôlée.

\section{Faire mi-chemin plutôt que faire tout le trajet ou ne pas partir du tout}

Dans leur «discussion" Webster et al. ont noté que "l'etendue de l'application par les médecins et le personnel infirmier de l'ordre de non réanimation avec limitation du traitement était généralement imprévue ". Uls ont recommandé que l'ordre de non réanimation ne doit pas être considéré comme équivalent à l'ordre de retrait de toute therapie. Ceci mérite d'être souligné d'une façon importante. Afin de diminuer les dangers d'illogisme, de confusion et de mauvaise compréhension entre les équipes de médecins et de personnel infirmier en rotation dans l'hôpital, il est crucial que le dossier du patient indique clairement lesquelles des interventions doivent être exclues. Il pourrait être utile que "les directives de procédure » requièrent que les buts du traitement et les désirs du patient et/ou de sa famille soient écrits dans le dossier. De telles directives écrites sont d'une grande valeur au personnel en charge d'une urgence et peuvent éviter que le patient subisse des interventions inappropriees. ${ }^{2.4}$

\section{Le patient preneur de décision}

Les auteurs n'étaient pas surpris de découvrir que 22/25 patients jugés inappropriés pour une réanimation cardiopulmonaire manquaient de capacité décisionnelle. Ils ont observé que "plusieurs membres du personnel infirmier ont exprimé le point de vue que l'ordre de non 
réanimation a été retardé et aurait pu être discuté plus tôt avec les patients quand leur état mental n'était pas sérieusement compromis ».

Il est décevant de noter que le protocole de non réanimation adopté dans ce rapport ne permet pas de consultation préalable avec le patient concemant de telles décisions excepté «quand l'évaluation clinique justifie la prescription d'un «ordre de non réanimation». A ce moment, la majorité des patients sont incapables d'exercer leurs droits à l'autonomie.

L'expérience de St. Michael n'est pas atypique dans les hôpitaux nord américains. Les décisions de non réanimation sont fréquemment entreprises tellement tardivement que la majorité des patients sont devenus incapables de participer au processus consultatif. ${ }^{5} \mathrm{Si}$ la participation du patient dans la décision d'une non réanimation est importante, alors il est nécessaire de trouver les moyens efficaces afin d'impliquer les patients avant qu'ils ne deviennent trop malades ou trop stupéfiés par des narcotiques pour exprimer leur propre désir. Ainsi, la proposition que de tels problèmes soient discutés avec tous les patients lors de leur admission à l'hôpital mérite une sérieuse considération.

\section{La famille comme délégué du pouvoir décisionnel}

Webster $e t$ al. ont noté que les médecins se questionnent quelquefois sur "l'ambivalence familiale " et que certains médecins, sont eux-mêmes incertains concernant le rôle "propre" des familles dans des décisions telles que que l'ordre de non réanimation ou de retrait de traitement. Quand les familles sont invitées à participer aux décisions de l'ordre de non réanimation, elles sentent « quelquefois qu'elles sont injustement placées pour prendre la décision si leur bien-aimé va vivre ou mourir ». Dans leurs analyses du processus d'application, les auteurs ont noté que les familles sont presque toujours consultées concernant les décisions de non réanimation. Cependant ils n'ont pas analysé la nature de cette consultation et ainsi, ils sont incapables d'expliquer si l'ambivalence familiale et le malaise étaient dûs au fait que la mauvaise question a été posée.

Peut-être le protocole doit être amandé afin que le point de vue familial soit scruté seulement s'il est impossible de reconstruire les propres désirs ou intentions du patient concernant le traitement. Ils doivent être consultés concernant le point de vue du patient, ses attitudes et ses valeurs plutôt que leur propre point de vue, attitudes et valeurs.

\section{Le rôle du personnel infirmier et auxiliaire dans les décisions de l'ordre de non réanimation}

Les auteurs ont indique qu'il y avait des différences importantes dans la perception et la communication entre les médecins et le personnel infirmier. Ils attribuent ces différences partiellement au fait que le médecin n'a pas consulté directement avec le personnel infirmier et partiellement à la propre perspective professionnelle du personnel infirmier issue du contact intense et prolongé avec le patient. ${ }^{7}$ Malgré les différences dans la perception entre les médecins et le personnel infirmier, les auteurs paraissent favoriser l'approche de groupe quant au processus décisionnel et considèrent que l'application de la politique de non réanimation a abouti à une meilleure communication aux soins intensifs. Ils n'encouragent cependant pas le personnel infirmier dans le rôle de défenseur du patient. Ceci est un point potentiel de conflit qui requiére plus d'analyses et d'évaluation.

Il serait intéressant de savoir quand les médecins consultent le personnel infirmier et s'ils considèrent le personnel infirmier comme source d'information sur le patient ou les désirs des familles, ou comme substitut au pouvoir décisionnel.

\section{Conclusion}

Webster et al. ont fourni une contribution importante à notre compréhension de la réalité du pouvoir décisionnel de l'ordre de non réanimation dans les hôpitaux canadiens. Ils concluent que d'autres études sont requises afin de corroborer leurs expériences positives. A ceci, on peut ajouter l'espoir que des investigations futures approfondiraient notre compréhension des valeurs sousjacentes au problème.

\section{Referencess}

1 Degner LF, Beaton JI. Life-Death Decisions in Health Care. Washington: Harper and Row, 1987; 78.

2 Younger SJ. Do not resuscitate orders: no longer secret, but still a problem. Hastings Center Report, 27, February, 1987.

3 Uhlmann RF, Cassel CK, McDonald WJ. Some treatment-withholding implications of no-code orders in an academic hospital. Crit Care Med 1984; 12: 879-81.

4 Evans $A L$, Brody B. The do-not-resuscitate order in teaching hospitals. JAMA 1985; 253: 2236-9.

5 Zimmerman JE, Knaus WA et al. The use and implications of do-not-resuscitate orders in intensive care units. JAMA 1986; 255: 351-6.

6 Bedell SE, Delbanco TL. Choices about cardiopulmonary resuscitation in the hospital. When do physicians talk with patients? N Engl J Med 1984; 310: 1089-93.

7 Lo B. The death of Clarence Herbert: withdrawing care is not murder. Ann Intern Med 1984; 101: 148-5. 